\title{
Online auction-based relay selection for cooperative communication in CR networks
}

\author{
Tao Jing ${ }^{1}$, Fan Zhang ${ }^{1}$, Wei Cheng ${ }^{2 *}$, Yan Huo $^{1}$ and Xiuzhen Cheng ${ }^{3}$
}

\begin{abstract}
Cognitive radio and cooperative communication are two new network technologies. So, the combination of these two new technologies is a novel solution to solve the problem of spectrum scarcity. Two main challenges exist in the integration of cognitive radio and cooperative communication. First, there is a lack of incentives for the participating wireless devices to serve as relay nodes. Second, there is not an effective relay selection policy. In this paper, we propose an online auction-based relay selection scheme for cooperative communication in cognitive radio (CR) networks. Specifically, we design an auction scheme through adopting stopping theory. The proposed scheme ensures that the primary user (PU) can effectively select a CR relay to transmit its packets in a given time bound. In addition, we have analytically proven the truthfulness and the individual rationality of our online auction scheme. Extensive simulations demonstrate that the proposed relay selection scheme can always successfully and efficiently select a proper relay for a PU and can achieve a higher cooperative communication throughput compared with the conventional schemes.
\end{abstract}

Keywords: Online auction; Cooperative relay selection; Optimal multiple stopping theory

\section{Introduction}

Cognitive radio technology has been recognized to be able to improve the spectrum utilization [1] through opportunistic spectrum access without interfering with the primary communications. Cooperative communication is an emerging technology that has the great potential to increase the throughput between two wireless devices [2] without requiring the support of infrastructure. Intuitively, the integration of these two hot technologies is expected to be a solution for the problems of spectrum scarcity and capacity limitation. In this paper, we particularly target on the scenario where the primary CR users (PU) seek to find a suitable cognitive radio (CR) relay to help transmit data, so that its end-to-end throughput can be increased and its end-to-end delay can be reduced.

Certainly, this hybrid cognitive-cooperative communication architecture is facing both technical and practical challenges. For instances, the relays should get sufficient incentives for participating in the cooperative communications. In addition, if there are multiple candidate relays,

*Correspondence: wcheng3@vcu.edu

2 Department of Computer Science, Virginia Commonwealth University, 23284 Richmond, VA, USA

Full list of author information is available at the end of the article it may be impossible/impractical for the primary user to collect information from all the candidate relays to make a decision of relay selection. The reason is twofold: (i) The time for collecting information from all the candidate relays may exceed the PU's end-to-end delay tolerance, and (ii) the availability of candidate relays may change during the collecting time as CR links are generally not stable. Therefore, an idea cognitive-cooperative scheme should be able to well incentivize the CR users and select a proper relay in a given time.

To design such a scheme, we conservatively consider to collect a candidate relay information and make the relay selection decision for this candidate relay online so that the collected information is valid and end-to-end delay requirement can be satisfied. In other words, the candidate relays send their information (including its sealed bid for cooperative transmissions) to the PU one by one. After receiving the information from a candidate relay, the PU immediately decides whether to choose this relay for cooperative transmission. Once a relay has been selected, the PU stops considering the rest of the candidate relays and pays the chosen relay a compensation based on its bid. In the paper, we consider the candidate relays as bidders and the PU as buyer, respectively.

\section{Springer}

(c) 2015 Jing et al:; licensee Springer. This is an Open Access article distributed under the terms of the Creative Commons Attribution License (http://creativecommons.org/licenses/by/4.0), which permits unrestricted use, distribution, and reproduction in any medium, provided the original work is properly credited. 
Specifically, we design an online auction-based relay selection scheme for cooperative CR communication. The main contributions of this paper can be summarized as follows:

1. We formulate the online relay selection problem as an optimal stopping problem and design an optimal stopping policy, which can guarantee the success of relay selection in a given time while control the cost of PU for utilizing cooperative communication. To the best of our knowledge, this is the first work to jointly adopt stopping theory and design auction scheme for relay selection in CR networks.

2. We analytically prove the truthfulness of the proposed online auction scheme without requiring any information about bidders behavior. This result guarantees the bid truthfulness of the candidate relays and thereby eliminates the possibility of market manipulation.

3. Our proposed online auction scheme is also individual rational. This feature indicates that both the PU and relay can be benefited from cooperative transmission, which guarantees the incentive of participation for both the PU and the relay.

4. Extensive experiments are conducted to study the performance of our online auction scheme. We investigate the impact of several parameters and compare our scheme with the optimal relay selection (ORS) scheme, which will look at all the candidate relays for minimizing the PU cost. The results demonstrate that the PU can always select an appropriate relay in the given time, and our scheme achieves a higher throughput than ORS with a tiny cost increase.

The rest of the paper is organized as follows: The related work is presented in Section 2. Preliminary is illustrated in Section 3. In Section 4, we describe the proposed stopping policy and the online auction scheme for cooperative communication. The analysis of our auction scheme appears in Section 5. The simulation results are reported and analyzed in Section 6. We conclude our paper in Section 7.

\section{Related work}

In this section, we briefly review the most related works to our research in the area of auction-based cooperative communication.

In [3], Huang et al. treated relay nodes as sellers and source nodes as buyers. Two auction mechanisms were proposed: the SNR auction and the power auction. In each auction mechanism, each user iteratively updates its bid according to the others' previous bids to maximize its own profit. The existence and the uniqueness of the Nash equilibrium in a single-relay network has been proven. In networks with multiple relays, the Nash equilibrium only exists under certain conditions. Zhang et al. considered the network scenario where there is a single relay and multiple user nodes in [4]. The authors formulated this problem as a sellers' market competition. A distributed algorithm has been developed to search the Nash equilibrium. In [5], Wang et al. studied the situation of one source node and multiple relay nodes. The authors modeled it as a Stackelberg game which is divided into two levels of hierarchy. The benefits of the source node and relay nodes were jointly considered. In [6], the authors formed a cooperative cognitive radio network (CCRN) where each PU employs a set of appropriate second users (SUs) for relaying the PU's information in an energy-efficiency solution. The relay selection and parameter optimization have been formulated as two Stackelberg games. A unique Nash equilibrium is achieved and proved for each game. In [7], the authors supposed that SUs can make rational choice between cooperation and band leasing. This novel CCRN model has been modeled as a Stackelberg game and analyzed. In [8], the impact of spectrum leasing was studied with a game-theoretic analysis. The authors first illustrated the significance of the problem with a two-secondary-user game. Subsequently, a $n$-secondaryuser game was studied and analyzed given the occupancy model of primary users. However, none of the above work had considered the truthfulness. As a result, these mechanisms are vulnerable to market manipulation and may produce poor outcome [9].

In [10], the authors considered the problem of cooperative spectrum sensing scheduling in a CR network. The author assumed that each SU has the freedom to decide whether or not to participate in cooperative spectrum sensing; if not, the SU can wiretap the decision on channel status made by other SUs. This mechanism strived the balance between conserving the energy for spectrum sensing and bring down the spectrum sensing performance. In [11], Yang et al. designed an optimal relay assignment algorithm for cooperative communication. In [12], Klemperer designed a double auction mechanism for cooperative communication. These two works satisfied the truthfulness requirements. They both needed to collect the information from all the source nodes and all the relay nodes, and their auction mechanisms were performed in a single-round fashion. As a comparison, our approach does not require the information from all relay nodes, and the auction is performed in an online manner.

\section{Preliminary}

In this section, we first give a description of online cooperative communication. Then, we present our system model and depict the two common economic properties (truthfulness and individual rationality) of an auction. 


\subsection{Online cooperative communication}

In a CR network, it often happens that a PU (source node) wants to send its data in a limited period of time. As the source node is far away from the destination, it needs to find a proper PU/SU as a relay to help it to transmit the data, as shown in Figure 1. The source node returns the relay node with monetary reward (the PU's payment to the selected node for relaying data). As the number of wireless users is rapidly increasing due to the fast growing number of mobile communication device and the source node needs to obey the delay constraint, it is impossible for the source node to detect all candidate relays. Besides, the state of candidate relays change quickly with time. So, after detecting a relay, the source node needs to make an online decision whether to adopt this relay or not. In this paper, we assume that the transmitter can only choose one relay for cooperative transmission. A work by Zhao et al. showed that it is sufficient for a source node to choose the best relay even when multiple relay nodes are available [13]. So, it is reasonable to assume that the PU transmitter will choose only one relay node for cooperative transmission.

There exist two types of cooperative communication pattern: amplify-and-forward (AF) and decode-andforward (DF) [2]. In AF, the relay amplifies the received data and transmits it to the destination. In DF, the relay fully decodes the data from the source node and then delivers it after re-encoding the data. Our proposed scheme can be applied to both of these two cooperative communication patterns.

\subsection{System model}

In this paper, we consider a time-slotted system consisted by a pair of primary transmitter and receiver (PU) and a number of $R$ candidate relays. The PU needs to send out a packet within $T$ time slots, and it values the packet by $\beta$. The time needed for collecting information from each relay is $\alpha$ time slots. Then, the PU can check at most $M=\lfloor T / \alpha\rfloor$ relays within $T$. The PU randomly collects information from the $R$ candidate relays one by one. The $i$ th candidate relay $X_{i}$ has a private true valuation $v_{i}$ and a bid price $b_{i}$ for forwarding the packet. When the PU collects the information from $X_{i}, X_{i}$ returns its bid $b_{i}$. Note that, the bid $b_{i}$ is valid for $\alpha$ time slots, and each candidate relay bids in a sealed-bid manner. After receiving $b_{i}$ from $X_{i}$, the PU needs to immediately make a decision on whether to select $X_{i}$ as the relay to transmit the packet or continue to check $X_{i+1}$. Table 1 summarizes the notations used through this paper.

\subsection{Economic properties \\ 3.3.1 Truthfulness}

An auction is truthful if revealing the private true value is the dominate strategy for each bidder. This means that no bidder can raise its utility by bidding $b_{i} \neq v_{i}$, no matter how others bids. This property can resist market manipulation and ensure the auction fairness and efficiency. In untruthful auctions, a selfish bidder can alter its bid to obtain extra outcome which will benefit itself but hurt the others.

\subsubsection{Individual rationality}

An auction is individual rational if a winner (the selected relay) is always paid by no less than its bid and the PU's cost is no more than the value of the packet. This property can ensure that the utilities of both the PU and the selected relay are no less than 0 . In other words, both the $\mathrm{PU}$ and the selected relay have incentive to participate in the cooperative communication.

\section{Online auction scheme for relay selection}

In this section, we formulate the relay selection process as an optimal stopping problem and present our proposed scheme in detail.

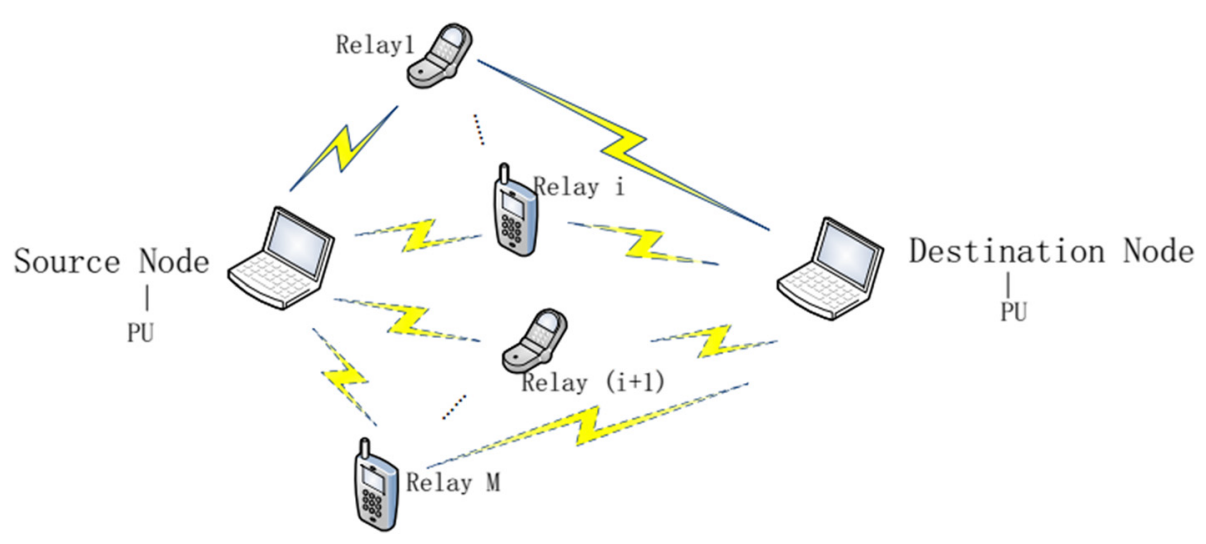

Figure 1 A model of online cooperative communication. 


\section{Table 1 Notations}

\begin{tabular}{ll}
\hline Notations & Definition \\
\hline$R$ & Number of candidate relays \\
$T$ & The delay tolerance for the PU \\
$\beta$ & The value of a packet \\
$\alpha$ & The time needed for checking each relay \\
$M$ & The maximal number of relays that can be checked within $T$ \\
$X_{i}$ & The ith candidate relay \\
$b_{i}$ & The bid of $X_{i}$ \\
$V_{i}$ & The private true valuation of $X_{i}$ \\
$Y_{n}$ & Real-valued reward function for checking $X_{n}$ \\
$V_{n}^{(M)}$ & Maximum reward at stage $n$ \\
$E\left(V_{n+1}^{(M)}\right)$ & Maximum expected reward at stage $n+1$ \\
$Z_{M-n}$ & A equal representation of $E\left(V_{M-n+1}^{(M)}\right)$ \\
\hline
\end{tabular}

\subsection{Problem formulation}

In the following, we first give a brief introduction of the finite stopping problems and then formulate the relay selection process accordingly.

Definition 1. Finite stopping problems: Given a sequence of random variables, $X_{1}, X_{2}, \ldots, X_{M}$, and a sequence of real-valued reward functions, $y_{0}(), y_{1}\left(X_{1}\right)$, $y_{2}\left(x_{1}, X_{2}\right), \ldots, y_{M}\left(x_{1}, x_{2}, \ldots, X_{M}\right)$, the objective is to find a $n$, so that the reward function $y_{n}\left(x_{1}, x_{2}, \ldots, X_{n}\right)$ is the maximal. Note that the joint distribution of the random variables and the reward functions are known and that $X$ denotes the random variable and $x$ denotes the value of a random variable, respectively.

In order to maximize the PU's utility, the PU makes each decision by comparing the instantaneous reward of selecting $X_{i}$ with the expected reward of checking $X_{i+1}$. Accordingly, we formulate the relay selection process as a sequence of decision problem which is defined based on the theory of optimal stopping [14] below.

According to [15], the PU knows (or can learn) the distribution of the bidding price of the bidders (candidate relays). We assume that there is no collusion among the candidate relays, and the bidding price of the candidate relays is independently distributed. In this paper, we particularly assume the bidding price follows the normal distribution with the mean value $\mu$ and the standard deviation $\sigma$, i.e., $f\left(b_{i}\right)=\frac{1}{\sqrt{2 \pi} \sigma} e^{-\frac{\left(b_{i}-\mu\right)^{2}}{2 \sigma^{2}}}$, where $f\left(b_{i}\right)$ is the probability density function of $b_{i}$. Note that, our proposed scheme can be generally applied to any bidding price distribution.
We divide the bidding price range into $J$ intervals so that the price is within a finite-state space. We use $S$ to represent the finite-state space of the bidding price.

$$
S=\left\{s_{1}=0, s_{2}, s_{3}, \ldots, s_{J}=\beta, s_{J+1}=+\infty\right\} .
$$

If the bidding price $b_{i} \in\left[s_{j}, s_{j+1}\right)$, we say that $b_{i}$ is in state $s_{j}$. The probability that $b_{i}$ is in state $s_{j}(j=1,2, \ldots, J)$ is:

$$
p_{j}=\int_{s_{j}}^{s_{j+1}} f\left(b_{i}\right) d b_{i}=\int_{s_{j}}^{s_{j+1}} \frac{1}{\sqrt{2 \pi} \sigma} e^{-\frac{\left(b_{i}-\mu\right)^{2}}{2 \sigma^{2}}} d b_{i} .
$$

We use $X_{n}$ to denote the bidding price of the $n$th checked candidate relay. As a result, the probability that $X_{n}$ bid for $s_{j}$ is:

$$
P\left(X_{n}=s_{j}\right)=p_{j}, 1 \leq j \leq J, 1 \leq n \leq M .
$$

Note that, in order to ensure that the PU's cost is no larger than $\beta$, we set $s_{J}=\beta$ and $s_{J+1}=\infty$.

Considering the fact that the time consumed for checking candidate relays are also valuable to the PU (because the PU can use them to transmit other packets, and the PU has a time limit to transmit a packet), checking more candidate relays should result in less PU's utility. Therefore, we use a decreasing function $f(n)=C^{\frac{n \cdot \alpha}{T}}$ to represent the time's impact, where $C \in(0,1]$. Accordingly the PU's realvalued reward function for checking the $n$th candidate relay is:

$$
\begin{aligned}
Y_{n}= & y_{n}\left(X_{1}, X_{2}, \ldots, X_{n}\right)=\left(\beta-X_{n}\right) \cdot f(n) \\
= & \left(\beta-X_{n}\right) \cdot C^{\frac{n \cdot \alpha}{T}} \\
& \quad \text { for } 1 \leq n \leq M, C \in(0,1] .
\end{aligned}
$$

According to [14], a necessary condition for the existence of optimal stopping rules to solve the problem is that $Y_{n}$ should satisfy the following two requirements:

$$
\begin{aligned}
& E\left\{\sup _{n} Y_{n}\right\}<+\infty . \\
& \limsup _{n \rightarrow+\infty} Y_{n} \leq Y_{+\infty} .
\end{aligned}
$$

We have proven that the $Y_{n}$ defined in (3) satisfies the requirements. Due to the page limit, we will report the proof in our technical report.

\subsection{Online auction-based selection scheme}

We use $V_{n}^{(M)}\left(x_{1}, x_{2}, \ldots, x_{n}\right)$ to represent the maximum reward that could be obtained after checking $n$ candidate relays:

$$
\begin{aligned}
V_{n}^{(M)}\left(x_{1}, x_{2}, \ldots, x_{n}\right)= & \max \left\{y_{n}\left(x_{1}, x_{2}, \ldots, x_{n}\right),\right. \\
& E\left(V_{n+1}^{(M)}\left(x_{1}, x_{2}, \ldots, x_{n}, X_{n+1}\right)\right. \\
& \left.\left.\mid X_{1}=x_{1}, X_{2}=x_{2}, \ldots, X_{n}=x_{n}\right)\right\} .
\end{aligned}
$$


In the above equation, $y_{n}\left(x_{1}, x_{2}, \ldots, x_{n}\right)$ means the instantaneous reward if we stop at stage $n, \quad E\left(V_{n+1}^{(M)}\left(x_{1}, x_{2}, \ldots, x_{n}, X_{n+1}\right) \mid X_{1}=x_{1}, X_{2}=x_{2}, \ldots\right.$, $\left.X_{n}=x_{n}\right)$ represents the expected maximum reward by proceeding to stage $n+1$. Thus, it is better for the PU to stop checking the next candidate relay when the instantaneous reward is larger than the expected maximum reward. Accordingly, the stopping rule is:

$$
\begin{aligned}
& \text { If: } y_{n}\left(x_{1}, x_{2}, \ldots, x_{n}\right) \geq E\left(V_{n+1}^{(M)}\left(x_{1}, x_{2}, \ldots, x_{n}, X_{n+1}\right)\right. \\
& \left.\mid X_{1}=x_{1}, X_{2}=x_{2}, \ldots, X_{n}=x_{n}\right) \text {, }
\end{aligned}
$$

the PU stops at stage $n$ (selects the $n$th candidate relay); otherwise, it continues to check the next candidate relay.

We use the method of backward induction to solve the finite stopping problem under the above stopping rule. Since the PU has to stop at stage $M$, we first calculate the optimal stopping rule at stage $M-1$, then conclude the optimal rule at stage $M-2$, and so on until we get the optimal rule at stage 1 . We use $Z_{M-n}$ to represent the maximum expected reward at stage $n+1$, i.e., $Z_{M-n}=E\left(V_{n+1}^{(M)}\right)$. We define that $Z_{0}=-\infty$. As the bidding price of each candidate relay is independent and identically distributed, the value of the $Z_{u}, u=M-n$, only depends on the remaining number of steps $u$. Therefore, the valuation of $Z_{u}$ can be calculated as follows:

$$
\begin{aligned}
Z_{1} & =E\left\{V_{M}^{(M)}\right\}=E\left\{\left(\beta-X_{M}\right) \cdot C^{\frac{M \cdot \alpha}{T}}\right\} \\
& =C^{\frac{M \cdot \alpha}{T}} \cdot\left(\beta-E\left(X_{M}\right)\right) \\
& =C^{\frac{M \cdot \alpha}{T}} \cdot \beta-C^{\frac{M \cdot \alpha}{T}} \cdot \sum_{j=1}^{J} s_{j} \cdot p_{j} .
\end{aligned}
$$

When $u \geq 1$, we can get:

$$
\begin{aligned}
Z_{u+1} & =\operatorname{Emax}\left\{Y_{M-u}, Z_{u}\right\} \\
& =\sum_{m} C^{\frac{(M-u) \cdot \alpha}{T}} \cdot\left(\beta-s_{m}\right) \cdot p_{m}+\sum_{n} Z_{u} \cdot p_{n},
\end{aligned}
$$

where the values of $m$ and $n$ need to satisfy the following condition:

$$
\begin{aligned}
& m \in\left\{j \mid C^{\frac{(M-u) \cdot \alpha}{T}} \cdot\left(\beta-s_{j}\right) \geq Z_{u}, j=1,2, \ldots, J\right\} \\
& n \in\left\{j \mid C^{\frac{(M-u) \cdot \alpha}{T}} \cdot\left(\beta-s_{j}\right)<Z_{u}, j=1,2, \ldots, J\right\}, \\
& m+n=J .
\end{aligned}
$$

Once a relay has been selected, the PU needs to calculate its payment to the selected relay. In order to frustrate the selfish candidate relays who bid untruthful, the payment that is paid to the winner should be bid-independent. As $Z_{u}$ is a static value for each $u \in[1, M]$, we can have the highest bidding price for the checked candidate relay to win at each step. Specifically, at step $n$, if $y_{n}\left(x_{1}, x_{2}, \ldots, x_{n}\right) \geq Z_{M-n}$, the winner gets payment $p_{n}$, which is calculated as follows:

$$
\begin{aligned}
& \text { As }: y_{n}\left(x_{1}, x_{2}, \ldots, x_{n}\right)=\left(\beta-p_{n}\right) \cdot C^{\frac{n \cdot \alpha}{T}}=Z_{M-n}, \\
& \text { So: } p_{n}=\beta-\frac{Z_{M-n}}{C^{\frac{n \cdot \alpha}{T}}} .
\end{aligned}
$$

Figure 2 briefly demonstrates the model of relay selection. Our proposed scheme is described in Algorithm 1. When the PU needs to send a packet in a given time, it first calculate $M$. The PU calculates $M$ based on (1) the delay requirement of a packet, (2) the time of checking each candidate relay, and (3) the expected transmission delay between the PU and the relays. After checking the $n$th candidate relay, the PU computes $y_{n}$ and the expected reward $Z_{M-n}$. According to the comparison of $y_{n}$ and $Z_{M-n}$, the PU makes a decision whether to select $X_{n}$ for cooperative communication immediately. If the PU does not chose any of the first $M-1$ candidate relay, it has to select the last candidate relay. Under this worst case, the PU needs to pay the $M$ th candidate relay's bidding price $b_{M}$. Note that, the decreasing function $f(n)$ can prevent the happening of the worst case, which has been verified by the simulations.

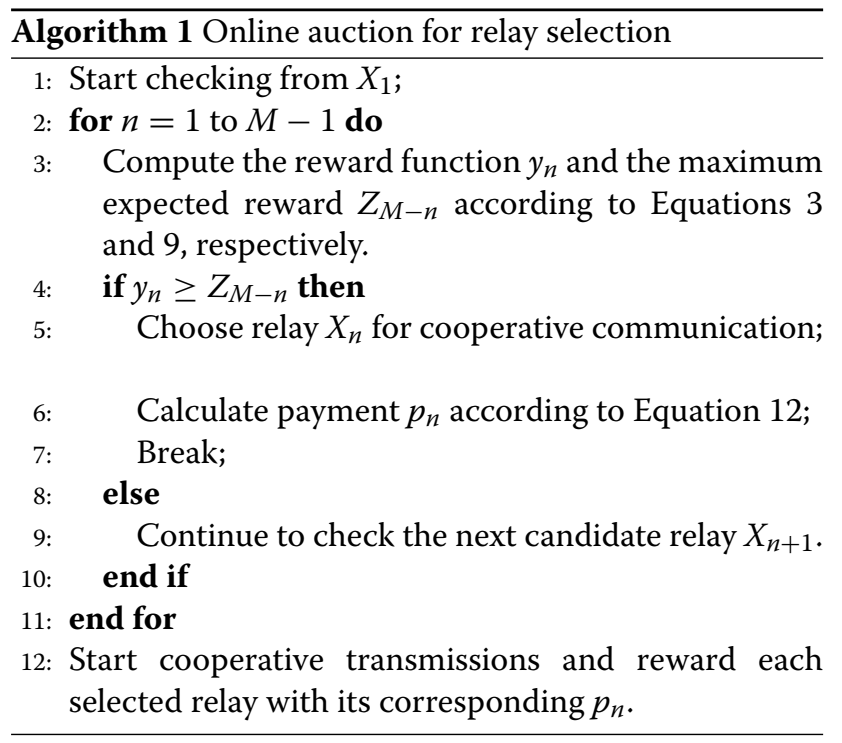

\section{Rationality and truthfulness analysis}

In this section, we justify that our proposed online auction-based relay selection scheme is both truthful and 


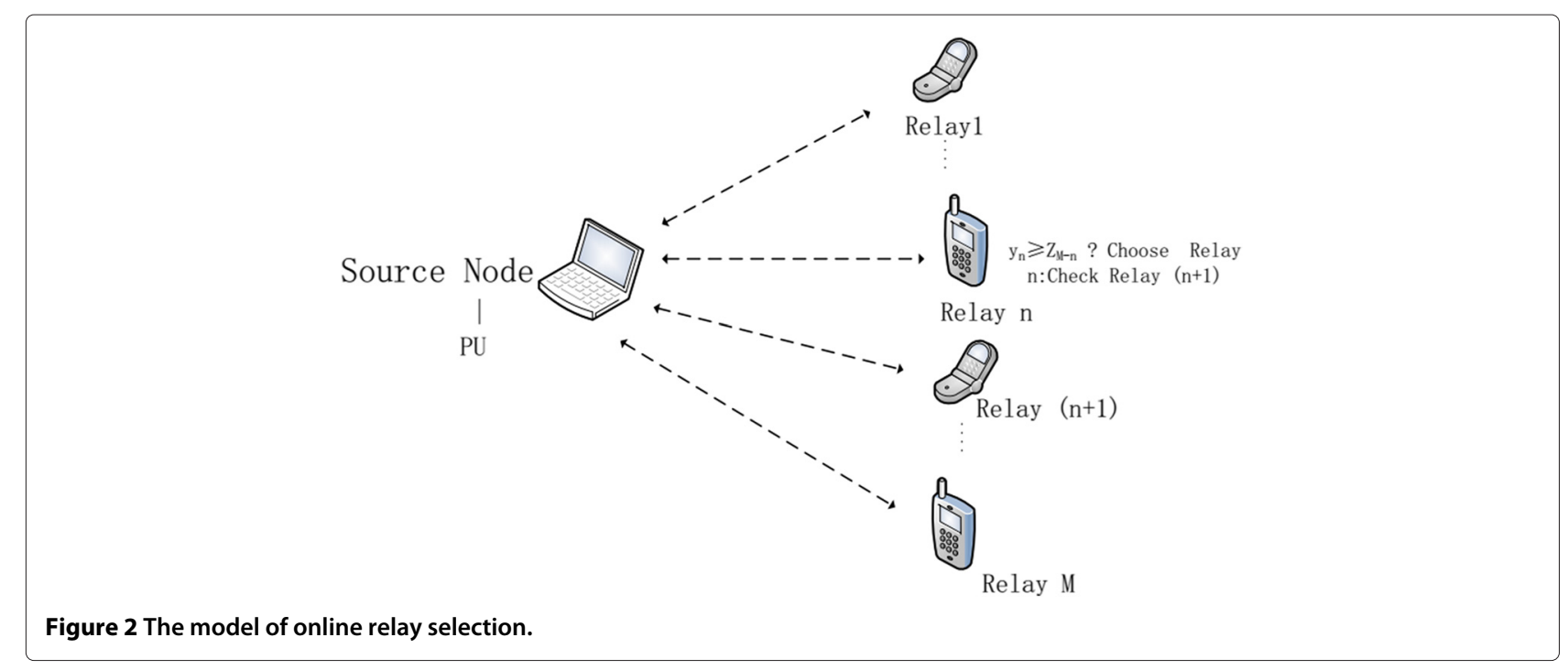

individual rational. Due to the page limit, we only present the main results. The detailed proof of the lemmas will be given in our technical report.

Lemma 1. $Z_{u} \geq 0$, for $u \in[1, M]$.

Theorem 1. The proposed online auction scheme is individual rational.

Proof. For winner $n, 1 \leq n \leq M-1$, the payment it can get is:

$$
\begin{gathered}
p_{n}=\beta-\frac{Z_{M-n}}{C^{\frac{n \cdot \alpha}{T}}} \\
\text { As: }\left(\beta-b_{n}\right) \cdot C^{\frac{n \cdot \alpha}{T}} \geq Z_{M-n} \\
\text { So: } b_{n} \leq \beta-\frac{Z_{M-n}}{C^{\frac{n \cdot \alpha}{T}}}=p_{n}
\end{gathered}
$$

We can get that utility $=p_{n}-b_{n} \geq 0$. If the winner is $M$, its utility $=0$. Therefore, we can conclude that, for any winner, its utility $\geq 0$.

For the PU, the value of the packet is $\beta$, and it has to pay $p_{n}$ to winner $n$. When $1 \leq n \leq M-1$ :

$$
\begin{aligned}
\text { utility } & =\beta-p_{n} \\
& =\frac{Z_{M-n}}{C^{\frac{n \cdot \alpha}{T}}}
\end{aligned}
$$

According to Lemma 1 , we have $Z_{u} \geq 0, u \in[1, M]$. Thus, utility $\geq 0$. When $n=M$, the utility $=\beta-b_{M}$. As the PU has to transmit the packet in this worst case, it is also rational.

Lemma 2. The payment to winner $n$ will be the same whatever it had bidden for $b_{n}$ or $v_{n}$.
Lemma 3. If bidder $n$ wins the auction when bidding truthfully, the payment from the $P U$ is no less than its evaluation, i.e., $p_{n} \geq v_{n}$.

Lemma 4. If bidder $n$ cannot win the auction when bidding truthfully, the possible payment from the PU is less than its evaluation, i.e., $p_{n} \leq v_{n}$.

Theorem 2. The auction scheme is truthful.

Proof. We use $u_{n}$ and $u_{n}^{\prime}$ to represent the utilities when bidder $n$ bids with $v_{n}$ and $b_{n}$, respectively.

I. We consider the case that $b_{n}>v_{n}$.

1. Bidder $n$ wins when bidding with either $b_{n}$ or $v_{n}$ : according to Lemma 2 and Lemma 3, we have $u_{n}^{\prime}=p_{n}-v_{n}=u_{n} \geq 0$;

2. Bidder $n$ loses with $b_{n}$ but wins with $v_{n}$ : according to Lemma 3, we can get $u_{n}^{\prime}=0 \leq p_{n}-v_{n}=u_{n}$;

3. Bidder $n$ wins with $b_{n}$ but loses with $v_{n}$ : this cannot happen in our auction scheme;

4. Bidder $n$ loses with both $b_{n}$ and $v_{n}: u_{n}^{\prime}=u_{n}=0$;

II. We consider the case that $b_{n}<v_{n}$.

1. Bidder $n$ wins when bidding with either $b_{n}$ or $v_{n}$ : according to Lemma 2 and Lemma 3, we have $u_{n}^{\prime}=p_{n}-v_{n}=u_{n} \geq 0$;

2. Bidder $n$ loses with $b_{n}$ but wins with $v_{n}$ : this cannot happen in our auction scheme;

3. Bidder $n$ wins with $b_{n}$ but loses with $v_{n}$ : according to Lemma 4 , we can get $u_{n}^{\prime}=p_{n}-v_{n} \leq 0=u_{n}$;

4. Bidder $n$ loses with both $b_{n}$ and $v_{n}: u_{n}^{\prime}=u_{n}=0$.

Through the analysis of all the possible cases, we can conclude that the utility of the bidder $n$ cannot be increased 
if $b_{n} \neq v_{n}$. Therefore, our auction scheme can ensure the bidding truthfulness.

\section{Simulations}

In this section, we conduct simulations to examine the performance of our proposed online auction mechanism. We assume that the bidding price of each candidate relay follows the normal distribution with the expected value $\mu=0.5$ and the variance $\sigma=0.2$. We partition the bidding price into $J=20$ intervals. We set the time limit for the PU to select a relay is 90 time slots, i.e. , $T=90$. In the following, we study the impact of the parameters such as $\alpha, C$, and $\beta$ on the number of observation steps and the reward of the PU, respectively. In order to justify the effectiveness of our scheme, we compare ours with the ORS scheme, which has the knowledge of who is the optimal relay that has the maximal PU reward among the $M$ candidate relays. In the ORS scheme, the PU checks the candidate relay one by one randomly and stops after checking the optimal relay. The simulations are conducted under several network scales. Particularly, the $M$ takes the value of 20,30, and 40, separately. Each reported result is the average of 100 independent instances.

\subsection{The impact of observation duration $\alpha$}

In this subsection, we set the time efficiency parameter $C=0.3$. The private value of the packet $\beta=0.9$. Figure 3 illustrates the relationship between the number of observation steps and the observation duration $\alpha$ under different network scales. From Figure 3, we observe that the PU generally stops checking within six steps in our scheme. When $\alpha$ changes from 2 to 2.2 , there is an obvious decline on the number of observation steps in our scheme. Generally speaking, a larger $\alpha$ results in a smaller observation steps. This is because the cost of checking a candidate relay is large when $\alpha$ is big. Thus, the PU intends to find a proper relay as soon as possible. On the other hand, when $\alpha$ is small, the PU may attempt to check more candidate relays to maximize its reward. We also find that the number of observation steps is always less than $M$, which indicates that the worst case of choosing the $M$ th relay will not happen. In addition, we can see that the number of observation steps of ORS is always higher than the corresponding one of ours. It means that our scheme needs less time for selecting a cooperative relay.

In Figure 4, we compare our scheme with ORS in terms of reward. Specifically, the reward of our scheme is normalized by the corresponding one of ORS, and we name this ratio as relative monetary reward. Figure 4 shows the relationship between the relative monetary reward of the PU and the observation duration. According to the results, we find that the relative reward of the PU fluctuates with the checking duration $\alpha$, but with a small variance (within 4\%), and that the relative monetary reward is always higher than $91.5 \%$. Jointly considering the results in Figure 3, we can conclude that our scheme selects the relay sooner, and the monetary reward for a single transmission is slightly smaller than the one of ORS.

By the time that the PU finds the optimal relay in OSR, the PU in our scheme can find multiple relays and get rewards via transmitting multiple packets. Therefore, to make a fair comparison in terms of reward, we define the ratio of monetary reward as the ratio between the total reward obtained in our scheme and the reward of

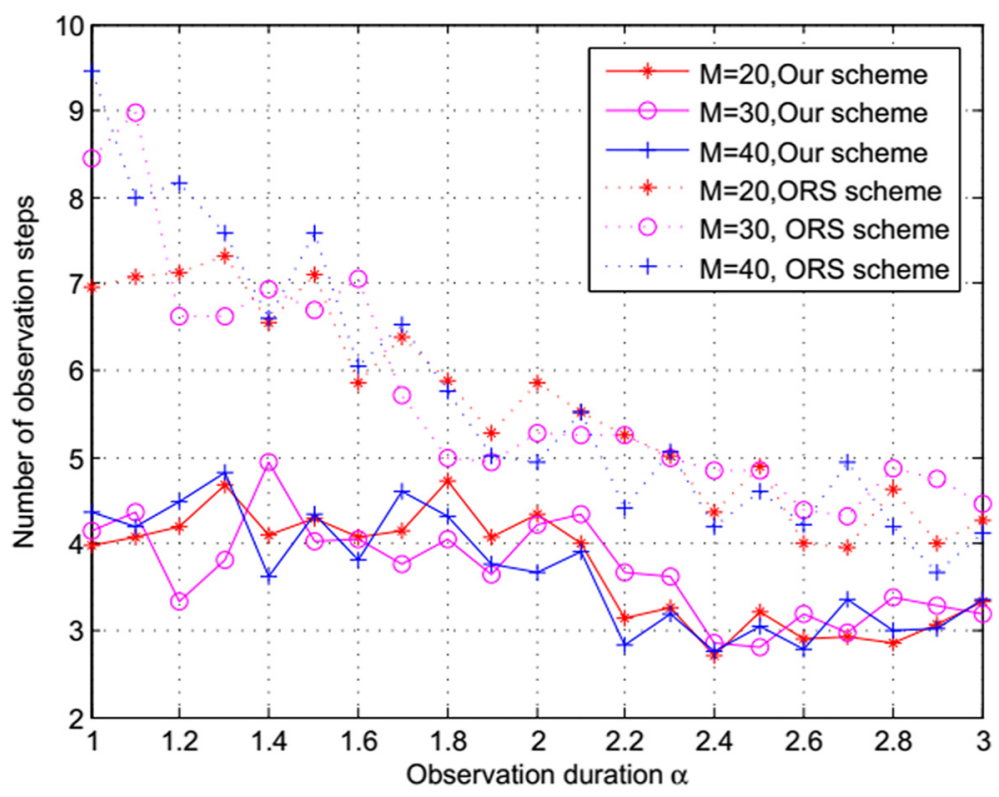

Figure 3 Average number of observation steps $\alpha$. 


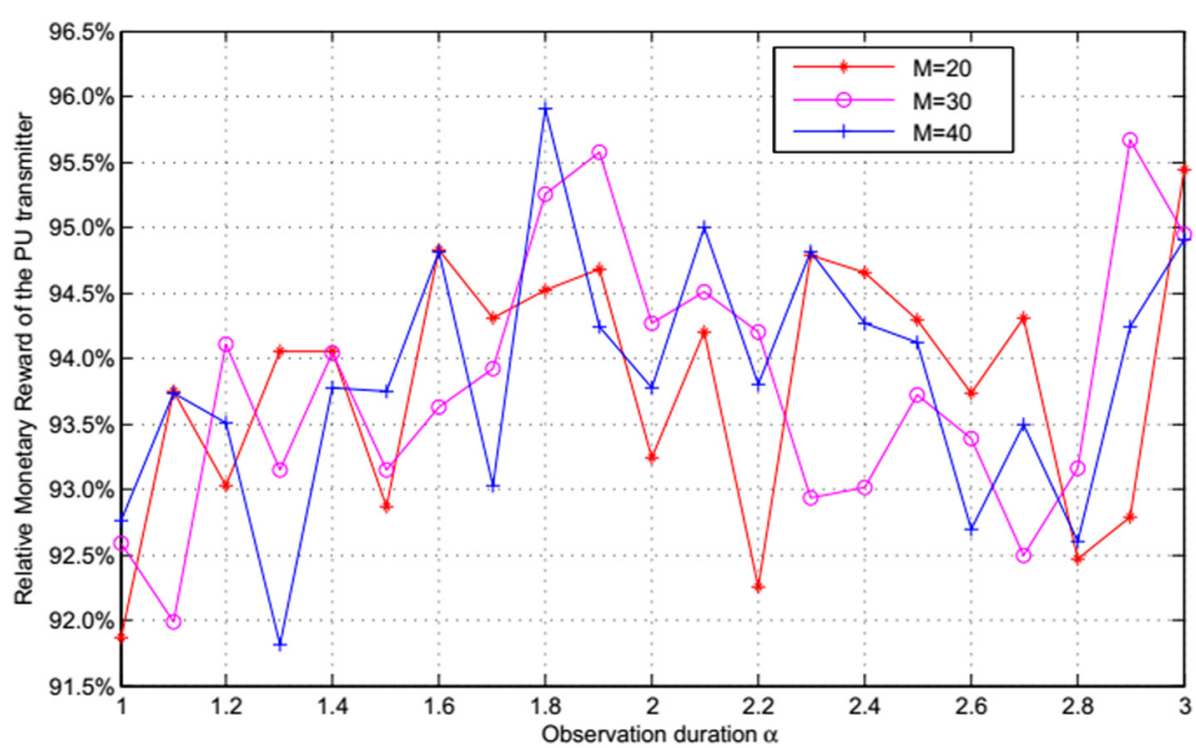

Figure 4 Relative reward for one packet transmission $\alpha$.

ORS during the same time period. The value of ratio is reported in the Figure 5. From Figure 5, we find that the ratio monetary reward is always higher than 1 . The maximum value can reach 2 when $\alpha=1$ and $M=40$. In other words, our proposed scheme can produce higher reward than OSR during the same time period.

\subsection{The impact of time efficiency $C$}

In this subsection, we set the observation duration $\alpha=2$, the private value of the packet $\beta=0.9$. From Figure 6, we can see that the PU can stop within six steps in our scheme, and the number of observation steps increases with the increase of $C$. This is because the impact of $C$ on the reward is big when $C$ is small. Thus, the PU intends to stop as soon as possible at a smaller $C$. By comparing with the ORS scheme, we can find that the number of observation steps in our scheme is always less than the corresponding one of ORS.

In Figure 7, we compare our scheme with ORS scheme in terms of relative reward for one packet transmission under different $C$. Similarly, the results of our scheme are normalized by the corresponding one of ORS. From Figure 7, we find that the relative reward decreases with the increase of $C$. The reason is that the time impact on the reward function is small when $C$ approximate 1 . In general, the relative reward is larger than $85 \%$.

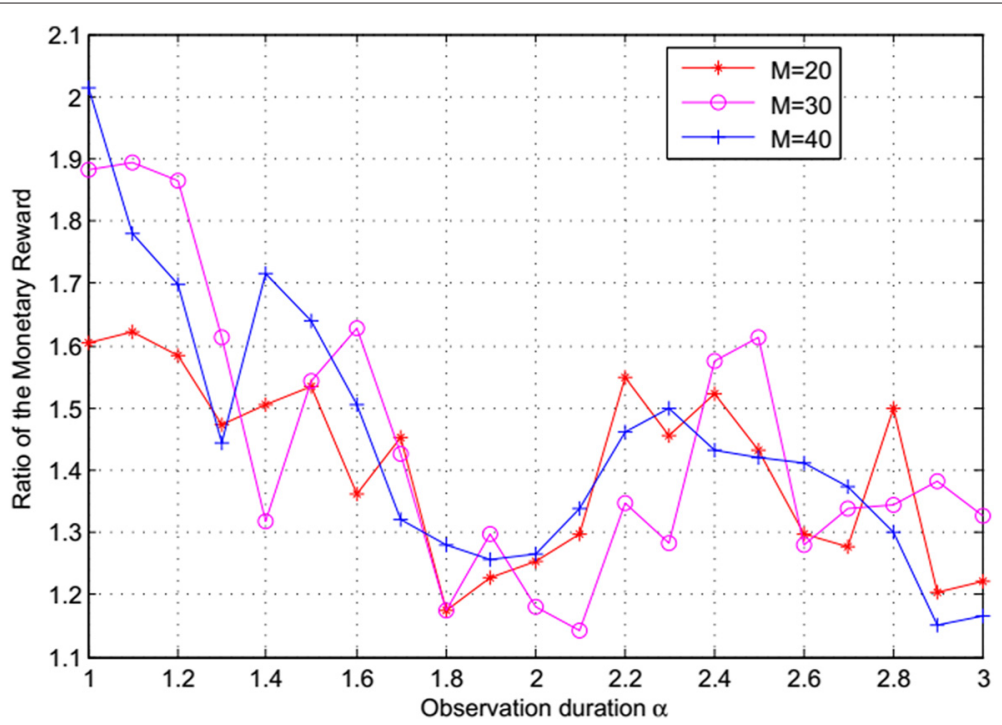

Figure 5 Ratio of reward during the same time period with $\alpha$. 


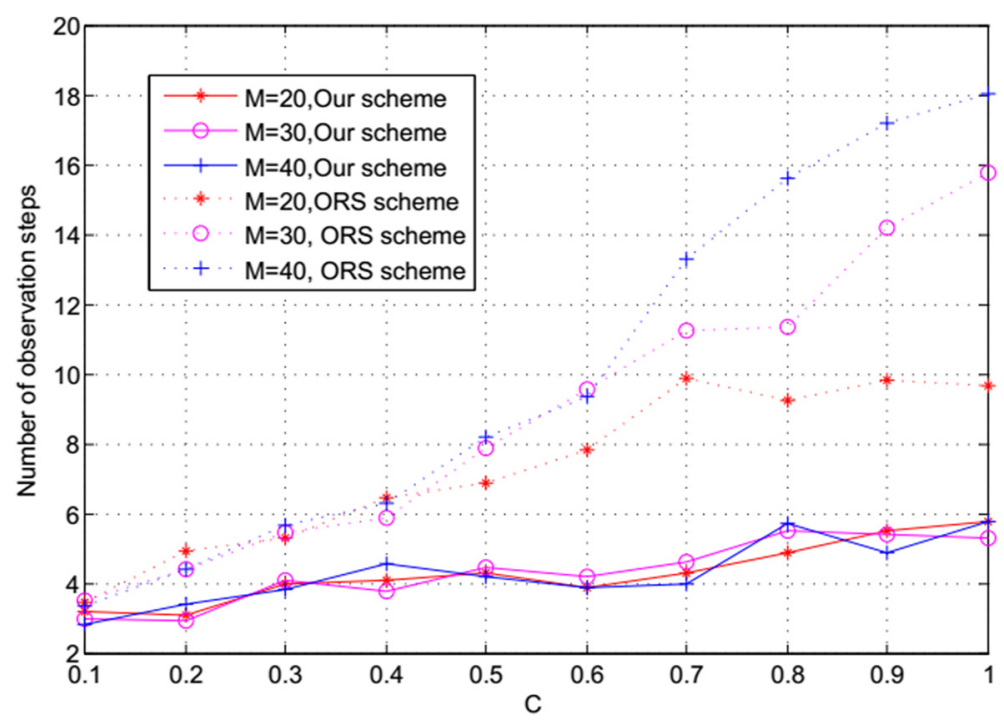

Figure 6 Average number of observation steps with $C$.

Similarly to the ratio results reported in Figure 5, we report the ratio of reward in Figure 8 under several $C$. From Figure 8, we can see that the ratio of monetary reward increases with the increase of $C$. The value of the ratio is always larger than 1 and can reach 3 when $M=40$ and $C=0.9$. In other words, the reward of our scheme is always higher than the one of ORS scheme during the same time period.

\subsection{The impact of private value $\beta$}

In this subjection, we set the observation duration $\alpha=$ 2 and time efficiency $C=0.3$. Figure 9 reports the relationship between the observation steps and $\beta$. From
Figure 9, we can observe that the number of observation steps decreases with $\beta$ at the beginning in our scheme. When $\beta$ is between 0.6 and 0.7 , the number of observation steps reaches the smallest value. Hereafter, the curve rises with the increase of $\beta$. When $\beta=1$, the number of observation steps reaches the largest value 5 . The reason for the trend of curve is that it is hard to find a relay that makes a small bid when the value of $\beta$ is small, and the PU intends to check more candidate relays to find larger reward when $\beta$ is big. We also observe that with different number of candidate relays, the number of observation steps almost stay the same. By comparing with ORS, we can see that the number of observation steps in

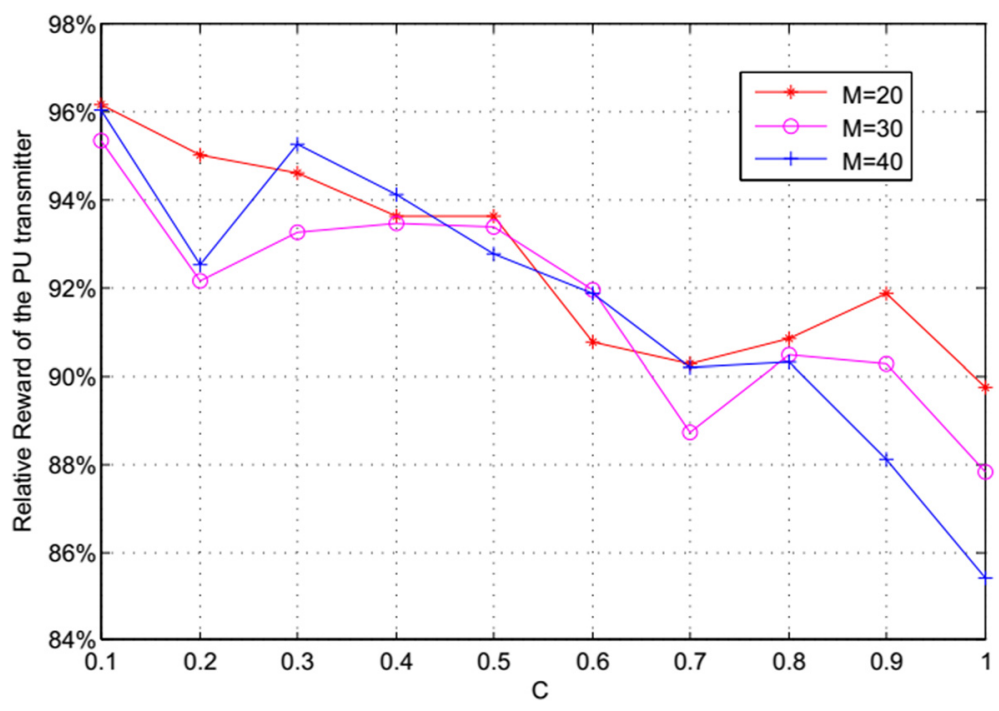

Figure 7 Relative reward for one packet transmission with $C$. 


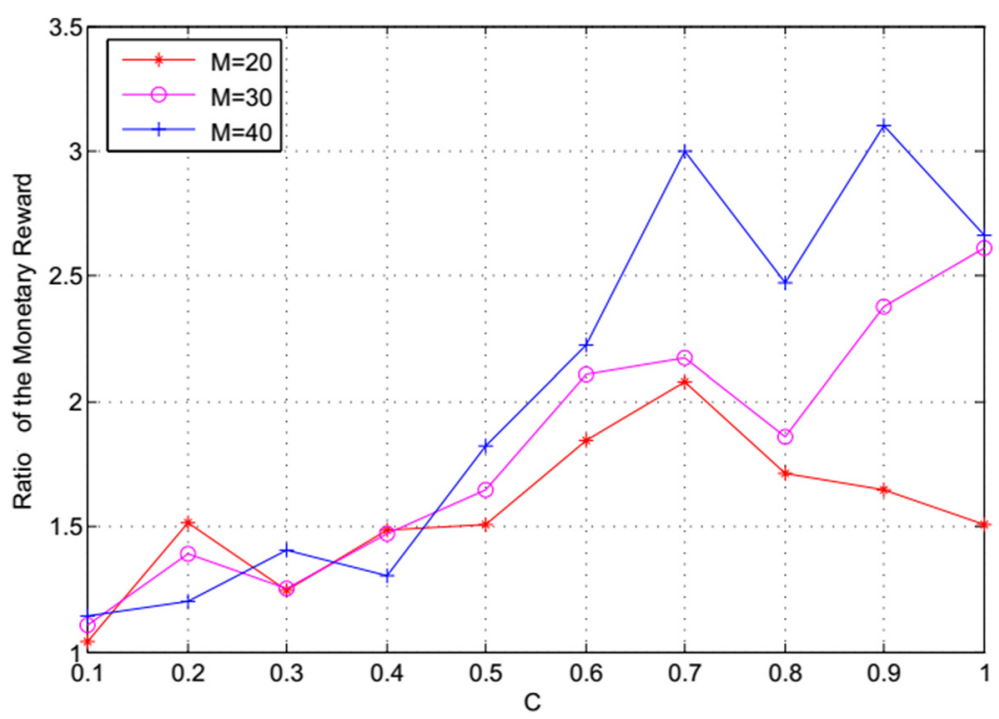

Figure 8 Ratio of reward during the same time period with $C$.

our scheme is always less than the corresponding one of ORS.

In Figure 10, we plot the relative reward for one packet transmission under several $\beta$. From Figure 10, we can see that the relative reward increases with the increase of $\beta$. The reason is that a larger $\beta$ can enlarge the reward. When $\beta$ is greater than 0.8 , the relative reward is larger than $90 \%$.

Figure 11 reports the ratio of reward time during the same time period under several $\beta$. From Figure 11, we can observe that the ratio fluctuates with $\beta$, but the value of the ratio is always higher than 1 .

In summary, we can conclude that the PU can always select a relay within six observations by our scheme, which is much less than the number of observation steps in OSR. Although the reward of transmitting one packet in our scheme may be slightly smaller than the corresponding one in OSR, the accumulated reward of our scheme is always much larger than the corresponding one of OSR for transmitting multiple packets in a given time period.

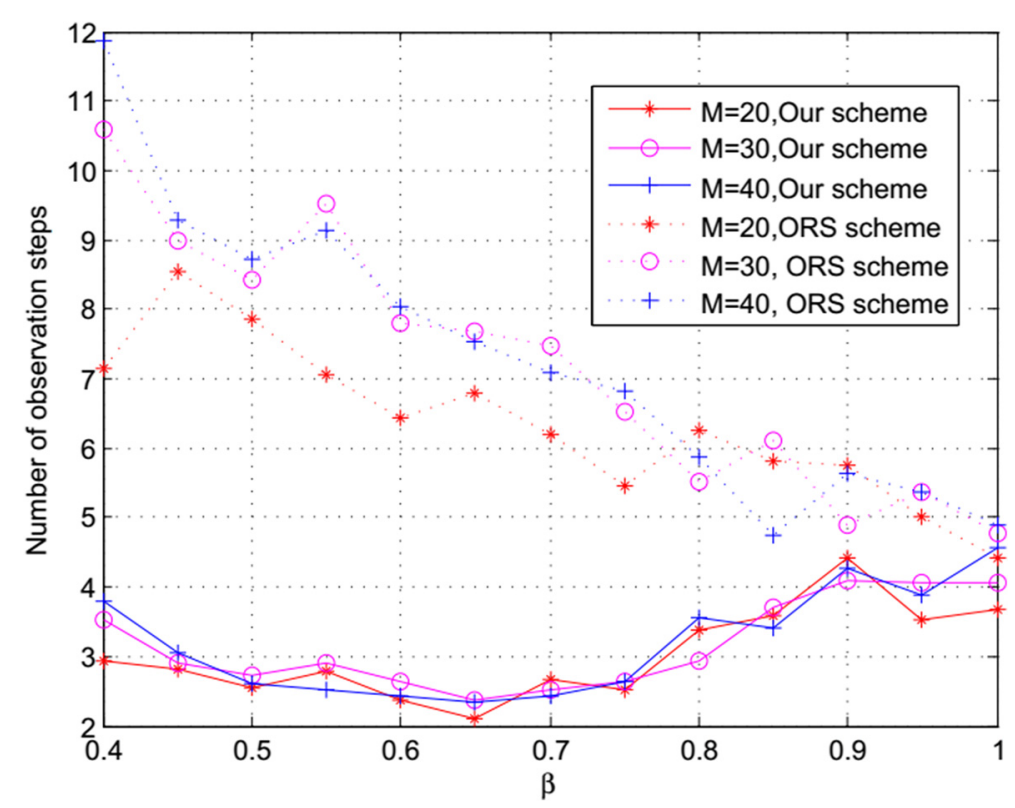

Figure 9 Average number of observation steps with $\beta$. 


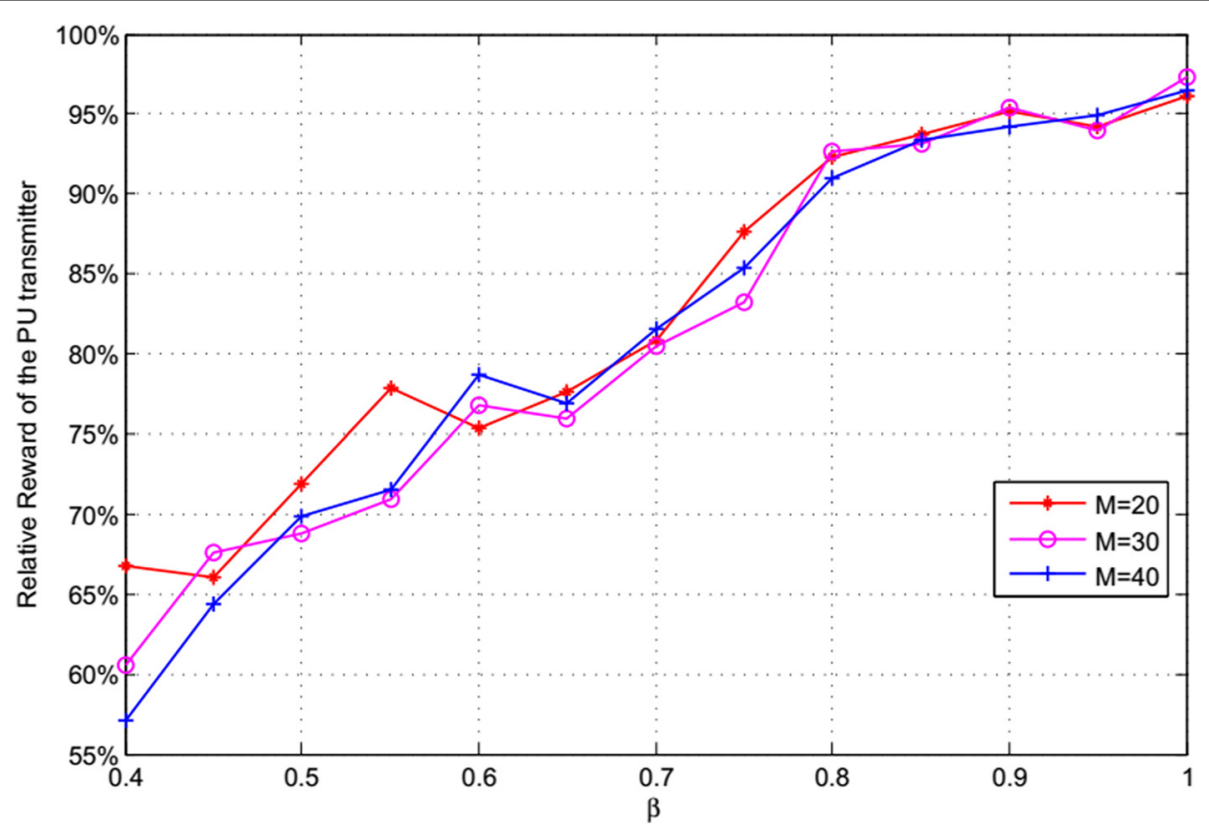

Figure 10 Relative reward for one packet transmission with $\beta$.

\section{Conclusions}

In this paper, we have designed an online auctionbased relay selection scheme for cooperative communication in CR networks. Without checking the information from all the candidate relays, the PU can select a desirable relay within a given time limit. Our scheme achieves the property of truthfulness, which assures that all the bidders reveal their true valuations and eliminates the fear of market manipulation. Meanwhile, our scheme is individual rational, which guarantees that both the PU and relays can be benefited from cooperative communication. Extensive simulations show that our scheme enables the PU to select a proper relay in a very short time and achieves a higher accumulated reward in a given time period comparing to the OSR scheme.

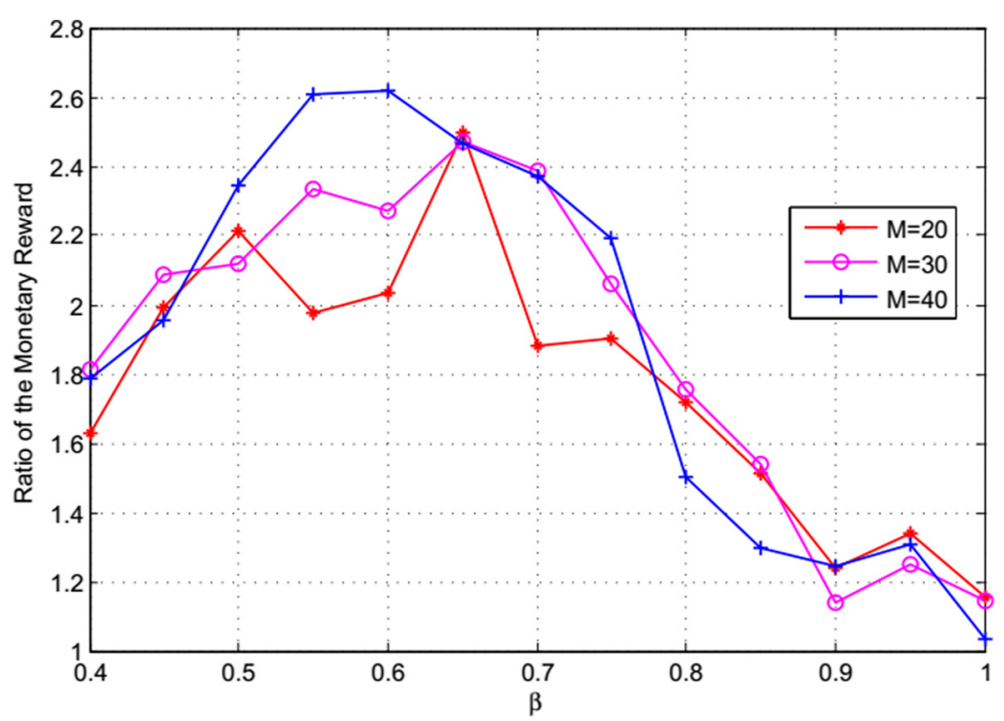

Figure 11 Ratio of reward during the same time period with $\beta$. 


\section{Competing interests}

The authors declare that they have no competing interests.

\section{Acknowledgements}

This work is partially supported by the National Natural Science Foundation of China (Grant Nos. 61272505, 61172074, and 61371069) and the National Science Foundation of the US (CNS-1265311).

\section{Author details}

${ }^{1}$ School of Electronics and Information Engineering, Beijing Jiaotong University, 100044 Beijing, China. ${ }^{2}$ Department of Computer Science, Virginia Commonwealth University, 23284 Richmond, VA, USA. ${ }^{3}$ Department of Computer Science, The George Washington University, 20052 Washington, DC, USA.

Received: 4 September 2014 Accepted: 7 January 2015

Published online: 03 February 2015

\section{References}

1. IF Akyildiz, WY Lee, MC Vuran, S Mohanty, NeXt generation/dynamic spectrum access/cognitive radio wireless networks: a survey. Comput. Networks. 50(13), 2127-2159 (2006)

2. JN Laneman, DN Tse, GW Wornell, Cooperative diversity in wireless networks: Efficient protocols and outage behavior. IEEE Trans. Inf. Theory. 50(12), 3062-3080 (2004)

3. J Huang, Z Han, M Chiang, Poor H, Auction-based resource allocation for cooperative communications. IEEE J. Sel. Areas Commun. 26(7), 1226-1237 (2008)

4. G Zhang, L Cong, L Zhao, K Yang, $\mathrm{H}$ Zhang, Competitive resource sharing based on game theory in cooperative relay networks. ETRI J. 31, 89-91 (2009)

5. B Wang, Z Han, KR Liu, in IEEE INFOCOM. Distributed relay selection and power control for multiuser cooperative communication networks using buyer/seller game (Barcelona, 6-12 May 2007), pp. 544-552

6. B Caoy, JW Mark, Q Zhang, R Lu, X Lin, XS Shen, in IEEE INFOCOM. On optimal communication strategies for cooperative cognitive radio networking, (2013), pp. 1774-1782

7. H Roh, C Jung, W Lee, DZ Du, in IEEE ICNC. A Stackelberg game for cooperative cognitive radio network with active SUs, (2013), pp. 911-915

8. H Roh, C Shao, S Lee, W Lee, DZ Du, in IEEE INFOCOM DEMO. Secondary user games with spectrum leasing market in cooperative cognitive radio networks, (2013), pp. 2461-2462

9. P Klemperer, What really matters in auction design. J. Econ. Perspect. 16, 169-189 (2002)

10. $\mathrm{H}$ Li, X Cheng, K Li, X Xing, T Jing, in IEEE INFOCOM. Utility-based cooperative spectrum sensing scheduling in cognitive radio networks, (2013), pp. 165-169

11. D Yang, X Fang, G Xue, HERA: An optimal relay assignment scheme for cooperative networks. IEEE J. Sel. Areas Commun. 30(2), 245-253 (2012)

12. P Klemperer, What really matters in auction design. J. Econ. Perspect. 16, 169-189 (2002)

13. Y Zhao, R Adve, TJ Lim, Improving amplify-and-forward relay networks: optimal power allocation versus selection. IEEE Trans. Wireless Commun. 6(8), 3114 (2007)

14. TS Ferguson, Optimal stopping and applications. http://www.math.ucla edu/ tom/Stopping/Contents.html

15. S Kern, SD Müller, N Hansen, D Büche, J Ocenasek, P Koumoutsakos, Learning probability distributions in continuous evolutionary algorithms-a comparative review. Nat. Comput. 3, 77-112 (2004)

\section{Submit your manuscript to a SpringerOpen ${ }^{\circ}$ journal and benefit from:}

- Convenient online submission

- Rigorous peer review

- Immediate publication on acceptance

- Open access: articles freely available online

- High visibility within the field

- Retaining the copyright to your article

Submit your next manuscript at $>$ springeropen.com 